\title{
The geometry of stellar motions in the nucleus region of the Ursa Major kinematic group
}

\author{
N. V. Chupina, V. G. Reva, and S. V. Vereshchagin \\ Institute of Astronomy of the Russian Academy of Sciences, 48 Pyatnitskaya Str., 109017 Moscow, Russia
}

Received 18 December 2000 / Accepted 15 February 2001

\begin{abstract}
The structure of the central part of the UMa stellar kinematic group was considered according to the data from Hipparcos, Tycho and ACT catalogues. We have shown that in the space occupied by the nucleus there are several populations of stars. The first includes ten stars which are very compactly located in the proper motion diagram and have a normal proper motion distribution. The second group includes six stars with large kinematic difference and there are six field stars placed here too. The question about their coexistence in a common region of space remains open. The well-known stars Mizar and Alcor appear to belong to the different subsystems and do not belong to a unique stellar system. The published radial velocities, as a rule, are determined with insufficient accuracy compared to the errors of the currently available proper motions. A high precision in the radial velocities measurements is necessary for a thorough study of the kinematics of stars inside UMa star system.
\end{abstract}

Key words. stars: formation - galaxies: open clusters: Ursa Major - stars: kinematics

\section{Introduction}

The Ursa Major stellar kinematic group (SKG) has been studied many times before. This group includes several tens of stars placed across almost the whole northern sky. However, there is a broad disagreement in the membership determinations. Thus, it includes 135 stars according to Roman (1949), 87 stars according to Eggen (1998) and 43 stars according to Soderblom \& Mayor (1993).

The UMa SKG as a stellar system is not uniform in space: a well determined nucleus is distinguished inside it and it includes up to 15 stars within the limits of $\alpha$ from $150^{\circ}$ to $220^{\circ}$ and $\delta$ from $50^{\circ}$ to $70^{\circ}$. We examine the sample of the nucleus stars with the membership determined in the abovementioned studies. This stellar system is placed relatively close to the Sun and proper motions of Hipparcos space astrometry mission (ESA 1997, here and after HIP) have accurate values, as a rule with an error less than 1 mas. The technique developed by us has allowed us to analyze separately the radial velocities and the proper motions. The separate consideration has the advantage of increased proper motion accuracy, which is lost after transition to the spatial velocities because of the smaller accuracy of the radial velocities.

Send offprint requests to: S. V. Vereshchagin, e-mail: svv@inasan.rssi.ru

\section{The data on the stars in the nucleus of UMa group}

Table 1 summarizes the identification numbers of stars belonging to the UMa SKG nucleus. The last three columns contain a 1 if the star belongs to UMa SKG nucleus according to Roman (1949), Soderblom \& Mayor (1993) and Eggen (1998). Otherwise, it contains a 0. We give a sample including 16 stars, but only for 11 of them do all three authors agree on membership. Table 2 presents some observational data for our sample of stars: the equatorial coordinates at J2000 equinox, stellar magnitudes in $V$ band, proper motions, parallaxes with their standard deviations from HIP (ESA 1997) and the radial velocities from the data in the Strasbourg Data Center catalogue 3190A (Duflot et al. 1995) with their standard deviations after Soderblom \& Mayor (1993).

The coordinates of the stars in the rectangular system centered at the Sun have the following limits (in pc): $x$ from -15 to $-4, y$ from 7 to 12 and $z$ from 19.5 to 24.5 pc. As usual, the $x$-axis is directed to the Galactic center, $y$ along the Galactic disk rotation and $z$ to the Galaxy North Pole. Thus, the nucleus has a shape stretched in the direction of the Galactic center and has a size of $11 \times$ $5 \times 5$ pc. Considering the Tycho Catalogue (ESA 1997, here and after TYC) and the ACT Reference Catalog (Urban et al. 1998) gave us six additional stars placed in the same space region. Table 3 contains their HIP/TYC data. These stars belong to the field based on the values of proper 
Table 1. The cross-identification of stars belonging to the UMa nucleus according to different catalogues and membership determinations by Roman (1949) - R, Soderblom \& Mayor (1993) - S and Eggen (1998) - E

\begin{tabular}{|c|c|c|c|c|c|c|c|c|c|c|}
\hline HIP & HD & Name & $\begin{array}{l}\text { Designation } \\
\text { in the } \\
\text { constellation }\end{array}$ & TYC & $\mathrm{ACT}$ & HR & FK5 & $\mathrm{R}$ & $\mathrm{S}$ & $\mathrm{E}$ \\
\hline 51814 & 91480 & & $37 \mathrm{UMa}$ & 381913721 & & 4141 & 398 & 1 & 1 & 1 \\
\hline 53910 & 95418 & Merak & $\beta \mathrm{UMa}$ & 382710791 & & 4295 & 416 & 1 & 1 & 1 \\
\hline 58001 & 103287 & Phecda & $\gamma \mathrm{UMa}$ & 383310341 & 1609350 & 4554 & 447 & 1 & 1 & 1 \\
\hline 59496 & 238087 & & & & & & & 0 & 0 & 1 \\
\hline 59774 & 106591 & Megrez & $\delta \mathrm{UMa}$ & 383710701 & & 4660 & 456 & 1 & 1 & 1 \\
\hline 61100 & 109011 & & & & & & & 1 & 1 & 0 \\
\hline 61481 & 109647 & & & 345819561 & 1610624 & & & 0 & 1 & 1 \\
\hline 61946 & 110463 & & & & 1833644 & & & 1 & 1 & 1 \\
\hline 62512 & 111456 & & & & & 4867 & & 1 & 1 & 1 \\
\hline 62956 & 112185 & Alioth & $\varepsilon \mathrm{UMa}$ & 384511901 & & 4905 & 483 & 1 & 1 & 1 \\
\hline 63503 & 113139 & & $78 \mathrm{UMa}$ & & & 4931 & & 1 & 1 & 1 \\
\hline 64532 & 115043 & & & & 1835486 & & & 1 & 1 & 1 \\
\hline 65327 & 238224 & & & & & & & 0 & 0 & 1 \\
\hline 65378 & 116656 & Mizar A & $\zeta \mathrm{UMa}$ & 385013851 & & 5054 & 497 & 1 & 1 & 1 \\
\hline 65477 & 116842 & Alcor & $80 \mathrm{UMa}$ & $\begin{array}{llll}3850 & 1384 & 1\end{array}$ & & 5062 & & 1 & 1 & 1 \\
\hline 69400 & 124752 & & & & 2027568 & & & 1 & 0 & 0 \\
\hline
\end{tabular}

Table 2. The observational astrometric parameters and radial velocities for the Table 1 stars

\begin{tabular}{|c|c|c|c|c|c|c|c|c|c|c|c|}
\hline HIP & \multicolumn{2}{|c|}{$\mathrm{J} 2000$} & mag & $\begin{array}{r}\mu_{\alpha} \\
\operatorname{mas} / \mathrm{yr}\end{array}$ & $\sigma_{\mu_{\alpha}}$ & $\begin{array}{r}\mu_{\delta} \\
\mathrm{mas} / \mathrm{yr}\end{array}$ & $\sigma_{\mu_{\delta}}$ & $\begin{array}{r}\pi \\
\text { mas }\end{array}$ & $\sigma_{\pi}$ & $\begin{array}{c}V_{\mathrm{r}} \\
\mathrm{km} \mathrm{s}^{-1} \\
\end{array}$ & $\begin{array}{c}\sigma_{V_{\mathrm{r}}} \\
\mathrm{km} \mathrm{s}^{-1}\end{array}$ \\
\hline 51814 & 103509.62 & +570457.2 & 5.16 & 65.65 & 0.45 & 37.11 & 0.38 & 37.80 & 0.61 & -10.4 & 0.5 \\
\hline 53910 & 110150.39 & +562256.4 & 2.34 & 81.66 & 0.37 & 33.74 & 0.38 & 41.07 & 0.60 & -12.0 & 0.5 \\
\hline 58001 & 115349.74 & +534141.0 & 2.41 & 107.76 & 0.48 & 11.16 & 0.50 & 38.99 & 0.68 & -12.6 & 0.5 \\
\hline 59496 & 121205.10 & +585535.0 & 10.05 & 101.96 & 0.97 & 20.26 & 1.01 & 35.24 & 1.24 & -15.0 & \\
\hline 59774 & 121525.45 & +570157.4 & 3.32 & 103.56 & 0.56 & 7.81 & 0.54 & 40.05 & 0.60 & -13.4 & 0.5 \\
\hline 61100 & 123118.81 & +550707.8 & 8.08 & 99.70 & 2.49 & -13.25 & 2.47 & 42.13 & 3.11 & -5.0 & \\
\hline 61481 & 123551.18 & +511317.3 & 8.52 & 113.00 & 0.84 & -8.06 & 0.76 & 38.08 & 1.11 & & \\
\hline 61946 & 124144.40 & +554328.9 & 8.27 & 121.53 & 0.65 & -4.36 & 0.63 & 43.06 & 0.82 & -6.3 & 0.3 \\
\hline 62512 & 124839.34 & +601911.6 & 5.83 & 107.79 & 2.65 & -30.60 & 3.08 & 41.39 & 3.20 & -12.0 & 1.5 \\
\hline 62956 & 125401.63 & +555735.4 & 1.76 & 111.74 & 0.45 & -8.99 & 0.49 & 40.30 & 0.62 & -9.3 & 0.5 \\
\hline 63503 & 130043.59 & +562158.8 & 4.93 & 108.36 & 0.50 & 2.67 & 0.51 & 40.06 & 0.60 & -9.8 & 0.5 \\
\hline 64532 & 131336.89 & +564229.9 & 6.82 & 111.91 & 0.56 & -17.88 & 0.65 & 38.92 & 0.67 & -8.9 & 0.1 \\
\hline 65327 & 132323.16 & +575422.2 & 9.69 & 127.49 & 1.63 & -4.82 & 1.34 & 39.84 & 1.44 & -6.6 & \\
\hline 65378 & 132355.42 & +545531.5 & 2.23 & 121.23 & 0.48 & -22.01 & 0.48 & 41.73 & 0.61 & -5.6 & 0.5 \\
\hline 65477 & 132513.42 & +545916.8 & 3.99 & 120.35 & 0.43 & -16.94 & 0.51 & 40.19 & 0.57 & -8.9 & 0.5 \\
\hline 69400 & 141226.83 & +673510.2 & 8.52 & 142.13 & 1.01 & -9.89 & 1.12 & 25.89 & 1.04 & -8.1 & \\
\hline
\end{tabular}

Table 3. The data on field stars in the nucleus spatial region. These stars do not belong to the UMa nucleus because they significantly differ in proper motions from the nucleus stars

\begin{tabular}{|c|c|c|c|c|c|c|c|c|c|c|c|}
\hline HIP & $\mathrm{HD}$ & TYC & \multicolumn{2}{|c|}{ J2000 } & $\begin{array}{c}V \\
\text { mag }\end{array}$ & $\begin{array}{c}\mu_{\alpha} \\
\mathrm{mas} / \mathrm{yr}\end{array}$ & $\sigma_{\mu_{\alpha}}$ & $\begin{array}{c}\mu_{\delta} \\
\text { mas } / y r\end{array}$ & $\sigma_{\mu_{\delta}}$ & $\begin{array}{c}\pi \\
\text { mas }\end{array}$ & $\sigma_{\pi}$ \\
\hline 56290 & 100203 & & 113220.76 & +610457.9 & 5.46 & -14.21 & 0.58 & -95.13 & 0.51 & 36.34 & 0.68 \\
\hline 56622 & 100831 & 383510591 & 113635.88 & $\begin{array}{lll}+56 & 08 & 08.1\end{array}$ & 7.16 & -178.35 & 1.06 & -92.25 & 0.98 & 22.92 & 1.36 \\
\hline 59432 & 105963 & 383410891 & 121127.93 & +532518.6 & 7.96 & -164.20 & 3.44 & -123.09 & 3.43 & 37.72 & 4.17 \\
\hline 60121 & & & 121948.23 & +524646.1 & 11.12 & -169.55 & 1.78 & -122.93 & 1.79 & 38.33 & 2.21 \\
\hline 61053 & 108954 & 384113031 & 123050.12 & +530434.2 & 6.20 & 19.46 & 0.50 & 182.10 & 0.47 & 45.58 & 0.62 \\
\hline & & 38292231 & 105429.3 & +575203.2 & 8.96 & -104.8 & 6.4 & -39.1 & 6.1 & 41.1 & 8.8 \\
\hline
\end{tabular}

motions. In this case the stars with $\sigma(\pi) / \pi<0.22$ were used.

All stars from Tables 1-3 are included in the HIP, with exception of the star 38292231 from the TYC.

\section{The transformation of proper motions into the coordinate system connected with the convergent point}

In Fig. 1 we show the distribution of proper motions for the stars from Table 2. The components of proper motions 


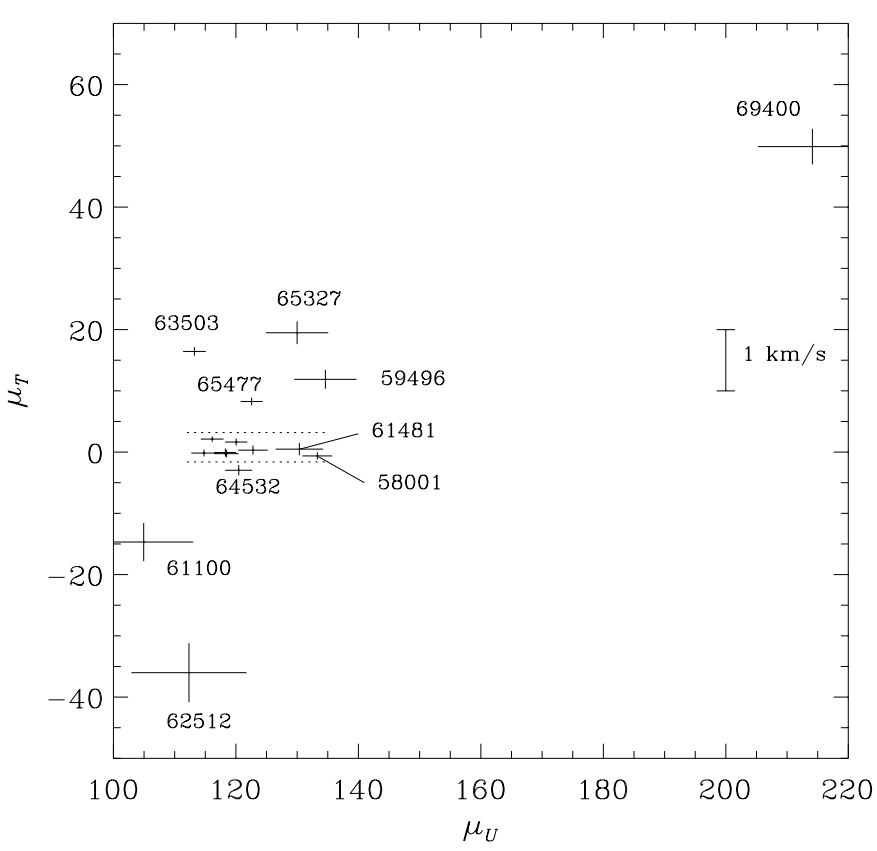

Fig. 1. The proper motion diagram for the stars selected as the nucleus members in the coordinate system connected with the convergent point (see Sect. 3). The identification numbers are given according to HIP near the points with the formal error bars. Between the dotted lines, the star sample satisfies the criterion for the normality of the $\mu_{\mathrm{T}}$-distribution (see Sect. 4)

were transformed to the coordinate system centered at each star and oriented to the convergent point. Following Kholopov (1981) and van Altena (1969) we calculated $\mu_{\mathrm{u}}$ as the proper motion component directed from the star to the convergent point and $\mu_{\mathrm{t}}$ as the component directed perpendicularly to $\mu_{\mathrm{u}}$ and with the positive direction to the North Pole. They were then corrected for the distances from the Sun and angular distances from the nucleus geometrical center: $\mu_{\mathrm{U}}=\mu_{\mathrm{u}} \frac{\sin \lambda_{\mathrm{c}}}{\sin \lambda} \frac{R}{R_{\mathrm{c}}}$ and $\mu_{\mathrm{T}}=\mu_{\mathrm{t}} \frac{R}{R_{\mathrm{c}}}$, where $R$ and $R_{\mathrm{c}}$ are the distances from the star and from the nucleus geometrical center to the Sun, $\lambda$ and $\lambda_{\mathrm{c}}$ are the angular distances from the star and from the nucleus geometrical center to the convergent point. We took $R_{\mathrm{c}}=20 \mathrm{pc}$ and $\lambda_{\mathrm{c}}=134.86$. Their values and errors are not of major importance because they are used for the normalization of the coefficients only. The coordinates of the convergent point $\left(A_{\text {conv }}, D_{\text {conv }}\right)$, necessary for the computation of $\mu_{\mathrm{U}}$ and $\mu_{\mathrm{T}}$ (Kholopov 1981; van Altena 1969), were determined by us as follows. Using initial $A_{\text {conv }}=305.0, D_{\text {conv }}-37^{\circ} .0$ (Allen 1973 ) we selected stars with small $\left|\mu_{\mathrm{T}}\right|$ and with the data on them calculated anew $\left(A_{\text {conv }}, D_{\text {conv }}\right)$ by means of the formulae described below. The iteration was repeated and we used the stars with $\left|\mu_{\mathrm{T}}\right| \leq 3$ mas; as one can see from Table 4 these are stars $51814,53910,58001,59774,61481,61946,62956,64532$, 65378 (here and further the star numbers are from the Hipparcos catalogue (ESA 1997)). We used the following
Table 4. The calculated proper motions and their errors in the coordinate system connected with the convergent point

\begin{tabular}{ccccc}
\hline HIP & $\begin{array}{c}\mu_{\mathrm{U}} \\
\text { mas/yr }\end{array}$ & $\sigma_{\mu_{\mathrm{U}}}$ & $\begin{array}{c}\mu_{\mathrm{T}} \\
\text { mas } / \mathrm{yr}\end{array}$ & $\sigma_{\mu_{\mathrm{T}}}$ \\
\hline 51814 & 114.79 & 2.07 & -0.15 & 0.56 \\
53910 & 116.13 & 1.86 & 2.13 & 0.45 \\
58001 & 133.30 & 2.44 & -0.62 & 0.60 \\
59496 & 134.61 & 5.08 & 11.88 & 1.54 \\
59774 & 118.28 & 1.74 & -0.06 & 0.66 \\
61100 & 104.95 & 8.08 & -14.68 & 3.12 \\
61481 & 130.36 & 3.87 & 0.49 & 1.02 \\
61946 & 122.79 & 2.40 & 0.32 & 0.74 \\
62512 & 112.34 & 9.40 & -36.01 & 4.82 \\
62956 & 118.48 & 1.97 & -0.25 & 0.61 \\
63503 & 113.23 & 1.86 & 16.44 & 0.70 \\
64532 & 120.46 & 2.19 & -2.96 & 0.84 \\
65327 & 130.00 & 5.09 & 19.49 & 1.86 \\
65378 & 120.05 & 1.81 & 1.65 & 0.58 \\
65477 & 122.57 & 1.78 & 8.26 & 0.63 \\
69400 & 214.14 & 8.90 & 49.88 & 2.90 \\
\hline
\end{tabular}

algorithm for the calculation of $\left(A_{\text {conv }}, D_{\text {conv }}\right)$. Using the space velocity components along the directions of $\mu_{\alpha}, \mu_{\delta}$ :

$V_{\alpha}=\frac{4.74047}{\pi} \mu_{\alpha}, \quad V_{\delta}=\frac{4.74047}{\pi} \mu_{\delta}$

we calculated the space velocity components for each star in the rectangular coordinate system, connected with the equatorial system:

$V_{x}=-V_{\alpha} \sin \alpha-\left(V_{\delta} \sin \delta-V_{\mathrm{r}} \cos \delta\right) \cos \alpha$,

$V_{y}=V_{\alpha} \cos \alpha-\left(V_{\delta} \sin \delta-V_{\mathrm{r}} \cos \delta\right) \sin \alpha$,

$V_{z}=V_{\delta} \cos \delta+V_{\mathrm{r}} \sin \delta$.

After the averaging of $\left(V_{x}, V_{y}, V_{z}\right)$ we calculate the equatorial coordinates of the convergent point:

$$
\begin{aligned}
& A_{\mathrm{conv}}=\arctan \frac{\left\langle V_{y}\right\rangle}{\left\langle V_{x}\right\rangle},\left\langle V_{z}\right\rangle \\
& D_{\mathrm{conv}}=\arctan \frac{\sqrt{\left\langle V_{x}\right\rangle^{2}+\left\langle V_{y}\right\rangle^{2}}}{} .
\end{aligned}
$$

The computed values are $A_{\text {conv }}=300.6864$ and $D_{\text {conv }}=$ -29.74465 (J2000).

The standard deviations of star positions in Fig. 1 were obtained taking into account the correlation coefficients $(\rho)$ between astrometric parameters $\mu_{\alpha}, \mu_{\delta}$, and $\pi$, see HIP (ESA 1997). The standard deviations of $\mu_{\mathrm{U}}$ and $\mu_{\mathrm{T}}$ were obtained from the equation (see ESA 1997):

$\operatorname{Cov}\left(\begin{array}{l}\mu_{\mathrm{U}} \\ \mu_{\mathrm{T}}\end{array}\right)=\mathrm{JCJ}^{\mathrm{T}}$

where $\mathrm{C}$ is the covariation matrix for $\mu_{\alpha}, \mu_{\delta}$ and $\pi$ :

$\mathrm{C}=\left(\begin{array}{ccc}\sigma_{\mu_{\alpha}}^{2} & \sigma_{\mu_{\alpha}} \sigma_{\mu_{\delta}} \rho_{\mu_{\alpha} \mu_{\delta}} & \sigma_{\mu_{\alpha}} \sigma_{\pi} \rho_{\mu_{\alpha} \pi} \\ \sigma_{\mu_{\alpha}} \sigma_{\mu_{\delta}} \rho_{\mu_{\alpha} \mu_{\delta}} & \sigma_{\mu_{\delta}}^{2} & \sigma_{\mu_{\delta}} \sigma_{\pi} \rho_{\mu_{\delta} \pi} \\ \sigma_{\mu_{\alpha}} \sigma_{\pi} \rho_{\mu_{\alpha} \pi} & \sigma_{\mu_{\delta}} \sigma_{\pi} \rho_{\mu_{\delta} \pi} & \sigma_{\pi}^{2}\end{array}\right)$ 


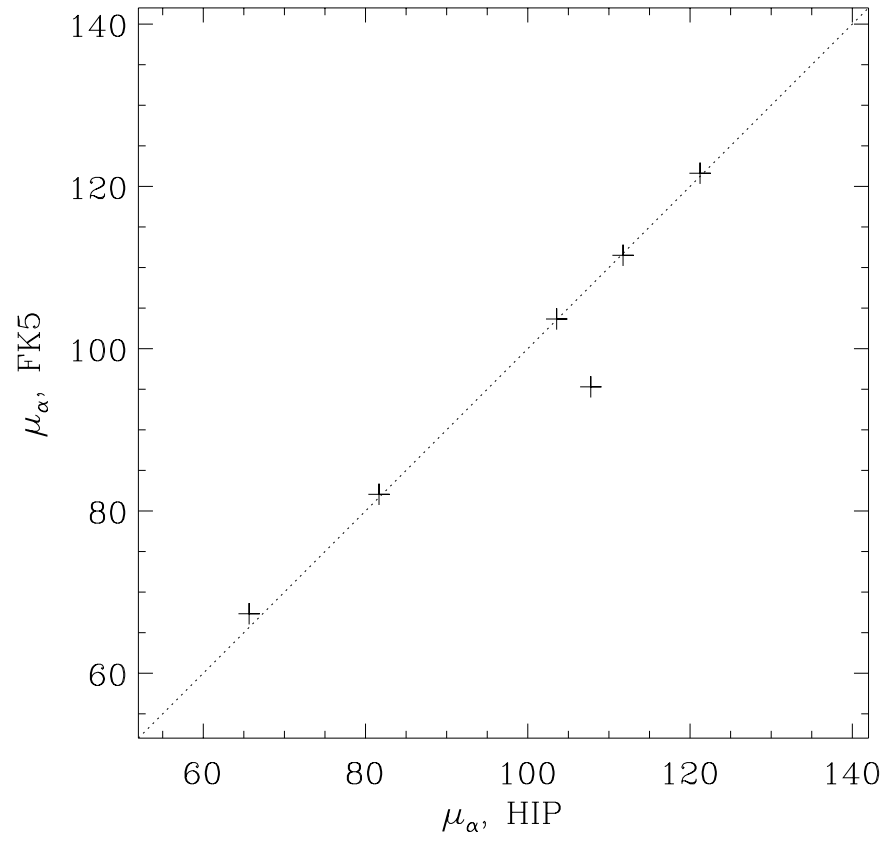

Fig. 2. The "HIP-FK5" right accession proper motion comparison. The star number 58001 jumped from dotted bisector

and $\mathrm{J}$ is the Jacobian of the $\left(\begin{array}{c}\mu_{\alpha} \\ \mu_{\delta} \\ \pi\end{array}\right)$ matrix transformation to the $\left(\begin{array}{l}\mu_{\mathrm{U}} \\ \mu_{\mathrm{T}}\end{array}\right)$. It is equal to

$\mathrm{J}=\left(\begin{array}{lll}\frac{\partial \mu_{\mathrm{U}}}{\partial \mu_{\alpha}} & \frac{\partial \mu_{\mathrm{U}}}{\partial \mu_{\delta}} & \frac{\partial \mu_{\mathrm{U}}}{\partial \pi} \\ \frac{\partial \mu_{\mathrm{T}}}{\partial \mu_{\alpha}} & \frac{\partial \mu_{\mathrm{T}}}{\partial \mu_{\delta}} & \frac{\partial \mu_{\mathrm{T}}}{\partial \pi}\end{array}\right)$,

and the terms of the Jacobian are, as one can see from the notation, the derivatives of the astrometric parameters. It is necessary to comment on some stars:

- The star 69400 was included in the nucleus by Roman (1949) only. It deviates from the average values of proper motions (see Fig. 1) and parallaxes (see Table 2). It has a very low index of chromospheric activity and was classified as a nonmember by Soderblom \& Mayor (1993). We also believe that it does not belong to the nucleus and we do not take it into account in our study;

- Star 58001. As is noticed by Eggen (1998), its $\mu_{\alpha}$, as given in the HIP, is not correct. From Fig. 2, where the comparison of the HIP proper motions with the proper motions from FK5 catalogue is displayed, it is easy to see that this is the case. If we use the value of $\mu_{\alpha}$ for this star from FK5, it moves leftward in the Fig. 1 to the region where most of the points are concentrated. In calculations below we used $\mu_{\alpha}$ from FK5;

- The star 61481 also may move to the left into the region of point concentration, as well as to the right, owing to a large error in $\pi$, see Table 2 .

\section{The $\mu_{\top}$ distribution structure}

It is possible to understand the shape of the distribution of the points along the $\mu_{\mathrm{T}}$-axis in Fig. 1 from the data given in Table 5. As noted before, we examine the proper motion diagram instead of the space velocities because of the low accuracy of the radial velocities. Table 5 provides the comparison of the observational $\mu_{\mathrm{T}}$ distribution and the normal one. Because of the small number of stars, we use the ratio of the range $R$ of $\mu_{\mathrm{T}}$ values to the $\mu_{\mathrm{T}}$ standard deviation $S$ as the normality criterion. In each new line of Table 5 we exclude from consideration one additional star having the outermost $\mu_{\mathrm{T}}$ value. The HIP number of the excluded star is in the first column of Table 5. The next columns contain the number of stars, $R / S$-relation and the upper and lower critical limits of $R / S$ necessary to satisfy normality at the $10 \%$ confidence level (Sachs 1972). In the case when the observational value of $R / S$ is confined between the tabulated critical limits and the distribution becomes normal, we place a flag equal to 1 in the last column of Table 5. In the opposite case the flag equals 0 . From Table 5 one can see that after exclusion of four stars with the numbers 62512 (the subsequent status of this star was changed, see Sect. 7), 65327, 63503 and 61100, our distribution becomes normal for the first time and after exclusion of yet three more stars (59496, 65477 and 64532 ) it finally becomes normal. Thus, our sample may be divided into two parts: the first has a normal $\mu_{\mathrm{T}}$-distribution and includes eight stars with numbers 51814, 53910, 58001, 59774, 61481, 61946, 62956 and 65378. Other stars do not belong to the normal general set. As one can see from Table 2, sometimes this may be a consequence of the large errors of the astrometric parameters; however, three stars: 63503, 64532, 65477 have errors smaller than 1 mas and are excluded not due to the large errors. It is necessary to note that, as we shall see, 64532 may be considered rather as a probable member according to its spectral features.

In the case when conditions of the equilibrium are satisfied in the UMa SKG nucleus and it is possible to consider $\mu_{\mathrm{T}}$-distribution as normal, the membership of the nucleus requires revision. According to the statistical procedure used, only eight stars with the high probability remain in the nucleus. Seven stars (including Alcor, 65477) represent possible nonmembers according to one critical parameter - proper motion. The status for stars 59496 , 61100,62512 , 65327 with large errors in the proper motions (see Table 4) may change with independent or more precise data (as will be done with 62512 in Sect. 7).

\section{The influence of the radial velocities errors}

We have developed a method allowing us to estimate simultaneously the convergence of proper motions and radial velocities. For this purpose we calculated the $A$ and $D$ coordinates for each star individually according to formulae (1), (2) and (3) without averaging. The $A-D$ diagram with the ellipses of errors is displayed in Fig. 3. The 
Table 5. The critical limits for the normal $\mu_{\mathrm{T}}$ distribution and kinematical membership status

\begin{tabular}{cccccccc}
\hline $\begin{array}{c}\text { The HIP number } \\
\text { of the excluding } \\
\text { star }\end{array}$ & $n$ & $R$ & $S$ & $R / S$ & \multicolumn{2}{c}{$\begin{array}{c}\text { r } / S \text { limits for 10\% } \\
\text { confidence level }\end{array}$} & $\begin{array}{c}\text { The nor- } \\
\text { mality } \\
\text { lower }\end{array}$ \\
\hline & 15 & 55.50 & $\begin{array}{c}13.06 \\
\text { Upper }\end{array}$ & 4.25 & 3.07 & 4.02 & 0 \\
62512 & 14 & 34.17 & 8.62 & 3.96 & 3.02 & 3.95 & 0 \\
65327 & 13 & 31.12 & 7.49 & 4.15 & 2.96 & 3.87 & 0 \\
63503 & 12 & 26.56 & 6.31 & 4.20 & 2.90 & 3.78 & 0 \\
61100 & 11 & 14.84 & 4.33 & 3.43 & 2.84 & 3.68 & 1 \\
59496 & 10 & 11.22 & 2.93 & 3.83 & 2.76 & 3.57 & 0 \\
65477 & 9 & 5.09 & 1.45 & 3.51 & 2.68 & 3.45 & 0 \\
64532 & 8 & 2.75 & 0.97 & 2.85 & 2.59 & 3.31 & 1 \\
53910 & 7 & 2.27 & 0.74 & 3.08 & 2.49 & 3.14 & 1 \\
65378 & 6 & 1.11 & 0.40 & 2.77 & 2.37 & 2.95 & 1 \\
58001 & 5 & 0.74 & 0.32 & 2.32 & 2.22 & 2.71 & 1
\end{tabular}

(Here 5 stars remain with numbers 59774, 51814, 61481, 61946 and 62956

with the smallest $\left|\mu_{\mathrm{T}}\right|$ values)

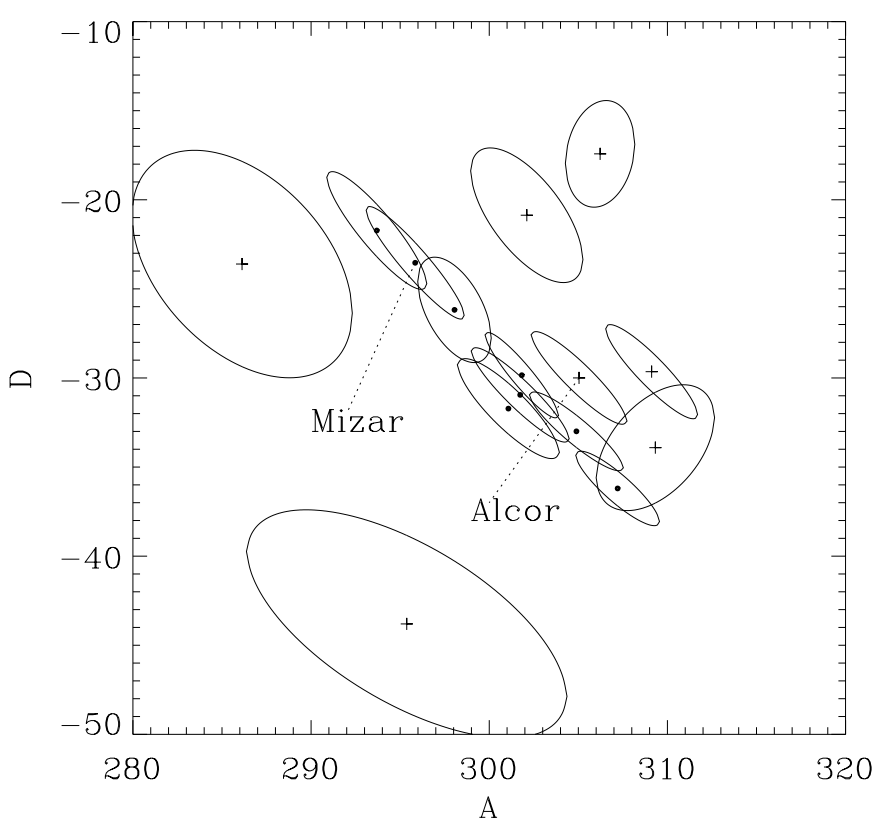

Fig. 3. The "apex points" diagram with the ellipses of errors. $A$ and $D$ - the equatorial coordinates of points where the space velocity vectors intersect the celestial sphere. The stars with high probability membership status on the basis of their normal $\mu_{\mathrm{T}}$-distribution are denoted by dots. The ellipses of errors correspond to the $3 \sigma$ confidence interval

values of $A$ and $D$ represent the equatorial coordinates of the point on the celestial sphere in which the space velocity vector intersects it. The ellipses of errors were calculated by means of the formulae which include the correlation coefficients between the astrometric parameters. In the calculations, we used the standard deviations equal to $\sigma_{V_{\mathrm{r}}}=0.3 \mathrm{~km} \mathrm{~s}^{-1}$ as the typical observational error (see Table 2) for radial velocities and $\sigma_{\mu_{\alpha}}, \sigma_{\mu_{\delta}}$ of each star individually. The major semi-axes of ellipses in Fig. 3 are approximately equal to three standard deviations due to radial velocities and $\mu_{\mathrm{U}}$ errors and minor semi-axes to three standard deviations due to $\mu_{\mathrm{T}}$ errors. The standard deviations of $A$ and $D$ were calculated as:

$\operatorname{Cov}\left(\begin{array}{l}A \\ D\end{array}\right)=\mathrm{JCJ}^{\mathrm{T}}$

with covariation matrix $\mathrm{C}$ :

$$
\mathrm{C}=\left(\begin{array}{cccc}
\sigma_{\mu_{\alpha}}^{2} & \sigma_{\mu_{\alpha}} \sigma_{\mu_{\delta}} \rho_{\mu_{\alpha} \mu_{\delta}} & \sigma_{\mu_{\alpha}} \sigma_{\pi} \rho_{\mu_{\alpha} \pi} & 0 \\
\sigma_{\mu_{\alpha}} \sigma_{\mu_{\delta}} \rho_{\mu_{\alpha} \mu_{\delta}} & \sigma_{\mu_{\delta}}^{2} & \sigma_{\mu_{\delta}} \sigma_{\pi} \rho_{\mu_{\delta} \pi} & 0 \\
\sigma_{\mu_{\alpha}} \sigma_{\pi} \rho_{\mu_{\alpha} \pi} & \sigma_{\mu_{\delta}} \sigma_{\pi} \rho_{\mu_{\delta} \pi} & \sigma_{\pi}^{2} & 0 \\
0 & 0 & 0 & \sigma_{V_{\mathrm{r}}}^{2}
\end{array}\right)
$$

and the Jacobian $\mathrm{J}$ for transformation from $\left(\begin{array}{c}\mu_{\alpha} \\ \mu_{\delta} \\ \pi \\ V_{\mathrm{r}}\end{array}\right)$ to $\left(\begin{array}{c}A \\ D\end{array}\right)$ equal to

$\mathrm{J}=\left(\begin{array}{llll}\frac{\partial A}{\partial \mu_{\alpha}} & \frac{\partial A}{\partial \mu_{\delta}} & \frac{\partial A}{\partial \pi} & \frac{\partial A}{\partial V_{\mathrm{r}}} \\ \frac{\partial D}{\partial \mu_{\alpha}} & \frac{\partial D}{\partial \mu_{\delta}} & \frac{\partial D}{\partial \pi} & \frac{\partial D}{\partial V_{\mathrm{r}}}\end{array}\right)$.

The following can be seen from Fig. 3:

1. There are two main effects. The ellipses of errors depend on the accuracy of the measurements of radial velocities and proper motions. On the other hand, the points are scattered along the diagonal from the upper left to the bottom right corner of the diagram (hereafter called "diagonal"). The latter effect may be caused by the dispersion of the internal stellar motions inside the system. Under assumption of the equality of the scatter of points in all directions, all points in the $A-D$ diagram would concentrate in a circular region;

2. The scatter of the points in the direction perpendicular to the diagonal is similar to the $\mu_{\mathrm{T}}$-distribution in Fig. 1: a high concentration of some points close to the diagonal and a location of other points distant from it; 
Table 6. The observed and corrected radial and space velocities of stars suspected to be in strong parallel spatial motion $\left(\right.$ in $\mathrm{km} \mathrm{s}^{-1}$ )

\begin{tabular}{ccccc}
\hline HIP & $\left(V_{\mathrm{r}}\right)_{\text {obs }}$ & $\left(V_{\mathrm{r}}\right)_{\text {corr }}$ & $V_{\text {obs }}$ & $V_{\text {corr }}$ \\
\hline 51814 & -10.4 & -11.9 & 14.06 & 15.17 \\
53910 & -12.0 & -11.5 & 15.75 & 15.35 \\
58001 & -12.6 & -10.4 & 17.17 & 15.66 \\
59774 & -13.4 & -9.8 & 18.18 & 15.70 \\
61946 & -6.3 & -9.3 & 14.80 & 16.32 \\
62956 & -9.3 & -8.6 & 16.14 & 15.76 \\
65378 & -5.6 & -7.7 & 15.08 & 15.97 \\
\hline
\end{tabular}

3. To get the equal sizes of the major and minor axes of the error ellipses, it is necessary to increase the accuracy of the determination of radial velocities. The analysis of the dependence between the length of a semi-axis and the error led us to the following conclusion. For precise HIP proper motions, the measurements of radial velocities require an accuracy better than $\sigma_{V_{r}}=0.1 \mathrm{~km} \mathrm{~s}^{-1}$. This value is significantly lower than the observational values given in Table 2 !

4. As it is shown by the numerical tests, the scatter of the points along the diagonal is dependent on the values of the moduli of radial velocities. As far as asymmetry is caused by radial velocities, it is possible to find (and then to check by observation) the new values of the radial velocities for all stars by minimization of the scatter of the points in the $A-D$ diagram (see Sect. 6 );

5. It is necessary to note that Mizar and Alcor in Fig. 3 lie far apart in the different regions of the diagram as well as in Fig. 1. The difference of their $V_{\mathrm{r}}$ values is larger than is possible for the components of a binary system.

\section{The correction of $V_{\mathrm{r}}$ for parallel space motion}

Now we consider the stars suspected to be in a strong parallel motion in space. These eight stars are situated very close to the diagonal in Fig. 3 and have the status of highly probable members in Table 5. They are listed in Table 6 with their observed space and radial velocities (with the exception of 61481 , because its radial velocity was not found). For these stars we have calculated the corrected values $\left(V_{\mathrm{r}}\right)_{\text {corr }}$ and $V_{\text {corr }}$ by minimization of the distance from the star "apex" $(A, D)$ in Fig. 3 to the convergent point $\left(A_{\text {conv }}, D_{\text {conv }}\right)$, see Sect. 3 . For this minimization we have for each star:

$\frac{\mathrm{d}}{\mathrm{d} V_{\mathrm{r}}}\left(\left(A-A_{\text {conv }}\right)^{2}+\left(D-D_{\text {conv }}\right)^{2}\right)=0$.

We have solved this equation numerically by the Newtonian method, changing the radial velocity step by step according to:

$\left(V_{\mathrm{r}}\right)_{\mathrm{corr}}=V_{\mathrm{r}}+\Delta V_{\mathrm{r}}$,
Table 7. Resulting nucleus membership determination with the spectroscopic membership indicator and some comments

\begin{tabular}{|c|c|}
\hline HIP & Comments \\
\hline \multicolumn{2}{|c|}{ Highly probable members (group 1 ): $\left|\mu_{\mathrm{T}}\right|<3.0$ mas } \\
\hline \multicolumn{2}{|l|}{59774} \\
\hline \multicolumn{2}{|l|}{51814} \\
\hline \multicolumn{2}{|l|}{62956} \\
\hline 61946 & $\begin{array}{l}\text { Included in UMa group by Soderblom \& } \\
\text { Mayor (1993) according to the chromo- } \\
\text { spheric emission value, CE - flag }=1\end{array}$ \\
\hline 61481 & $\mathrm{CE}-$ flag $=1$ \\
\hline 58001 & $\begin{array}{l}\mu_{\alpha} \text { is not correct in HIP and a value from } \\
\text { FK5 was used }\end{array}$ \\
\hline \multicolumn{2}{|r|}{ 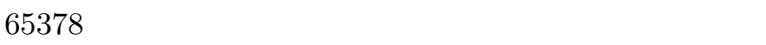 } \\
\hline \multicolumn{2}{|l|}{53910} \\
\hline 62512 & $\begin{array}{l}\mathrm{CE}-\text { flag }=1, \mu_{\delta}=-3.00 \mathrm{mas} / \mathrm{yr} \\
\text { was taken from PPM instead of } \mu_{\delta}= \\
-30.60 \mathrm{mas} / \mathrm{yr} \text { in HIP (see Table } 2)\end{array}$ \\
\hline 64532 & $\mathrm{CE}-$ flag $=1$, possible member \\
\hline \multicolumn{2}{|c|}{ Kinematically nonmembers (group 2): $\left|\mu_{\mathrm{T}}\right| \gg 3.0$ mas } \\
\hline \multicolumn{2}{|c|}{65477} \\
\hline 61100 & $\begin{array}{l}\text { CE - flag }=1 \text {, but suspected as spectro- } \\
\text { scopic binary, Soderblom \& Mayor (1993) }\end{array}$ \\
\hline \multicolumn{2}{|r|}{$e_{1}$} \\
\hline 69400 & $\begin{array}{l}\text { Excluded according to astrometry (see } \\
\text { Table 2) and small CE-index value } \\
\text { (Soderblom \& Mayor 1993) }\end{array}$ \\
\hline
\end{tabular}

where

$\Delta V_{\mathrm{r}}=-\frac{\left(A-A_{\text {conv }}\right) \frac{\mathrm{d} A}{\mathrm{~d} V_{\mathrm{r}}}+\left(D-D_{\text {conv }}\right) \frac{\mathrm{d} D}{\mathrm{~d} V_{\mathrm{r}}}}{\left(\frac{\mathrm{d} A}{\mathrm{~d} V_{\mathrm{r}}}\right)^{2}+\left(\frac{\mathrm{d} D}{\mathrm{~d} V_{\mathrm{r}}}\right)^{2}}$.

The radial velocities corrected in such a way give the mean value of the space velocity: $\left\langle V_{\text {corr }}\right\rangle=15.76 \mathrm{~km} \mathrm{~s}^{-1}$, $\sigma_{V_{\text {corr }}}=0.37 \mathrm{~km} \mathrm{~s}^{-1}$, whereas for the observed values we obtained: $\left\langle V_{\text {obs }}\right\rangle=15.95 \mathrm{~km} \mathrm{~s}^{-1}$ and $\sigma_{V_{\text {obs }}}=1.33 \mathrm{~km} \mathrm{~s}^{-1}$. One can see that the standard deviation has much decreased after correction. It means that the space velocity vectors became more uniform. Thus, if more precise observations would give in the future the radial velocities close to the corrected ones given in Table 6, the strong parallel movement of the stars belonging to the nucleus of the UMa group will be confirmed.

We now present our general impression about the space velocity distribution. The space motions of stars from our list (except 61481 without radial velocity data) are shown in Fig. 4. The $U, V, W$ components were calculated in usual way using the HIP proper motions, parallaxes and $V_{\mathrm{r}}$ from Table 2 . We note that our sample of highly probable members from Table 5 has a smaller point scatter than the entire sample. The distribution of points does not contradict the assumption that UMa SKG members have a common space motion in the direction of Galactic rotation - the scatter of the $V$-component is less than that of $W$ and approximately equals the $U$ 

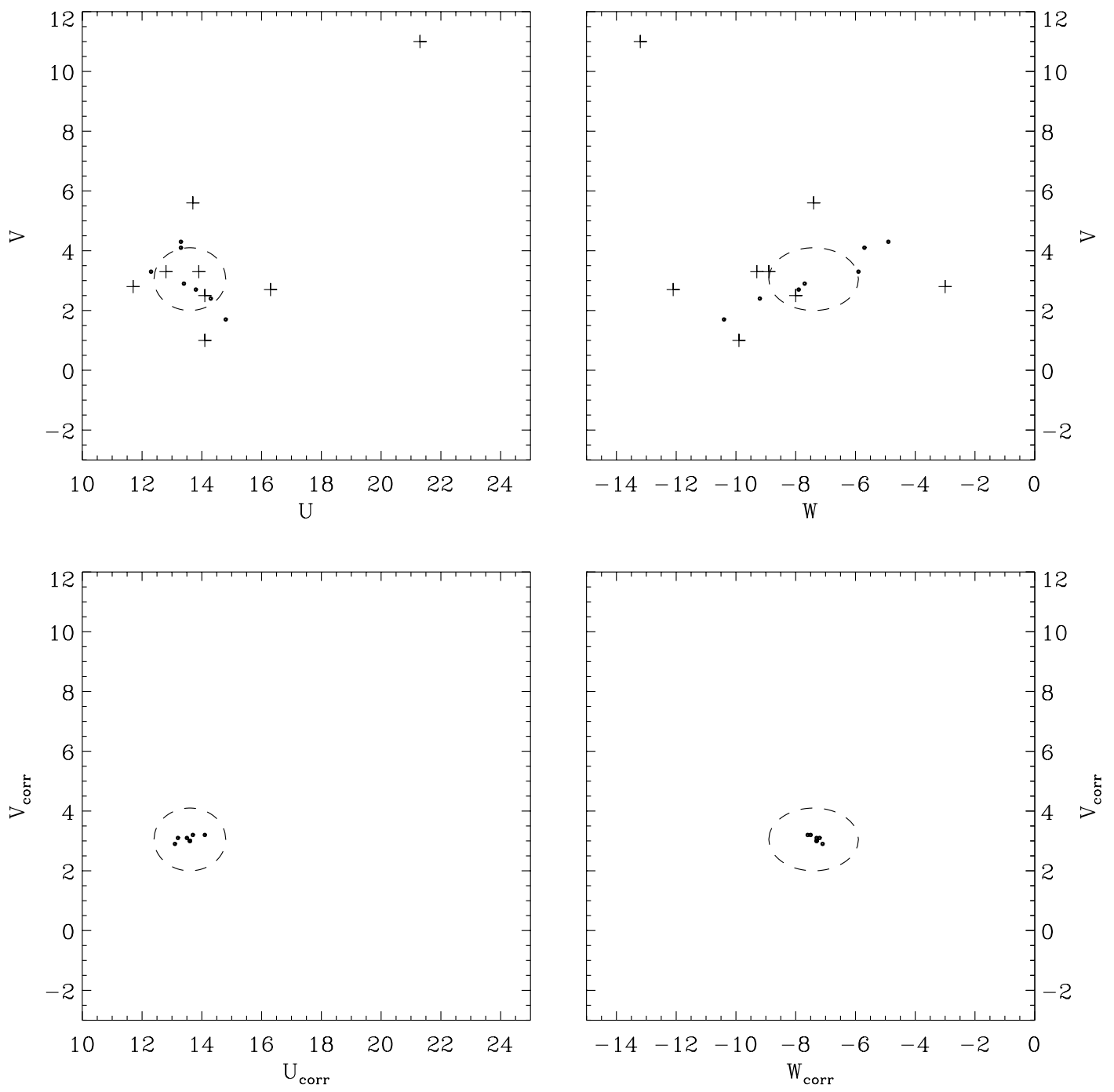

Fig. 4. Space motions of stars from our list and $V_{\mathrm{r}}$ correction results for highly probable members. Top panel is the $U, V, W$ distribution: dots denote highly probable kinematic members, crosses denote other stars from our list. Bottom panel gives the corrected velocity components $\left(U_{\text {corr }}, V_{\text {corr }}, W_{\text {corr }}\right)$ according to the procedure in Sect. 6 . The nonmember star 69400 lies far in the upper left corners of the top panels. The regions restricted by three mean errors of space velocity components are shown by the dashed line. They are centered at the mean point positions for highly probable members

scatter. The calculated dispersions have the values equal to $\sigma_{U}: \sigma_{V}: \sigma_{W}=0.8: 0.9: 2.0 \mathrm{~km} \mathrm{~s}^{-1}$. The mean errors of the components are $\left\langle\varepsilon_{U}\right\rangle:\left\langle\varepsilon_{V}\right\rangle:\left\langle\varepsilon_{W}\right\rangle=0.4: 0.35: 0.5 \mathrm{~km} \mathrm{~s}^{-1}$ (calculated from observation errors). They are considerably less than $\sigma_{U}, \sigma_{V}, \sigma_{W}$. In particular it means that some scatter in $V$-motions in UMa Group nucleus really exists. However, the large $V_{\mathrm{r}}$ errors do not allow us to construct an adequate picture for the true space motions. It confirms the bottom panel in Fig. 4: after $V_{\mathrm{r}}$ correction the highly probable members concentrate to a small region within the errors, which implies practicaly identical space velocities of the nucleus stars.

\section{Conclusions}

The final results concerning our membership determination are presented in Table 7 . A CE - flag $=1$ means that the star belongs to the UMa SKG according to a satisfactory value of the index of the strength of chromospheric emission (CE-index), see Soderblom \& Mayor (1993). Using the proper motion diagram shown in Fig. 1, the $A-D$ diagram in Fig. 3 and the results of Sect. 4, we have detected inside the nucleus region of the UMa SKG eight stars revealed as highly probable members in Table 5 and suspected in strong parallel motion with dispersion comparable with the lowest HIP errors (1 mas). These stars share a common space motion with a small dispersion of $1.33 \mathrm{~km} \mathrm{~s}^{-1}$. To this list we add two stars. The star 62512 was included after replacement of its $\mu_{\delta}$. As suspected by Eggen (1998), this $\mu_{\delta}$ has a large mistaken value in HIP. With the new $\mu_{\delta}$ from PPM (1991) the $\mu_{\mathrm{T}}$ became equal to $-2.67 \mathrm{mas} / \mathrm{yr}$, which fits into our best interval and the star transits to the highly probable members group. The star 64532 was included in the highly probable members group for two reasons: it has satisfactory value of the CE-index and $\left|\mu_{\mathrm{T}}\right|<3.0$, that do not 
corrupt the proper motion distribution (see Sect. 4). These two additional stars complete our list of highly probable members of the UMa nucleus presented in Table 7 as group 1. For some solar type stars, the probability is substantiated by the $\mathrm{CE}-$ flag $=1$. Thus, we arrive at the conclusion that in the central region of UMa SKG, ten stars are situated with strictly parallel vectors of spatial velocities.

The values of proper motions measured with high accuracy prevent from inclusion in the group 1 some other stars: 59496, 63503, 65477(Alcor). The stars 61100, 65327 have proper motion errors exceeding 1 mas (see Table 2), and their membership is questionable. 61100 has a $\mathrm{CE}-$ flag $=1$, which is a reason to include it in group 1 , but it is suspected to be a spectroscopic binary and the CE-index is not an adequate membership criterium. We have included these six stars into group 2 and believe that kinematically these stars do not belong to UMa SKG nucleus.

The well-known stars Mizar and Alcor belong to different groups and it is likely that they do not form a double system.

The asymmetry of the positions of the points in the $A-D$ diagram gives evidence that the published radial velocities need more precise measurements. The numerical method elaborated by us allows us, with the help of the $A-D$ diagram, to reduce the values of the radial velocities of selected stars to a quasi-parallel space motion of the whole group. If the new, more precise measurements of $V_{\mathrm{r}}$ give the values close to the values obtained by us as a result of correction, it would be a serious argument for a strong parallel motion of the eight selected stars.

Acknowledgements. We thank D. Soderblom for his useful remarks, L. R. Yungelson and A. E. Piskunov for reading the manuscript. Portion of this study was assisted by grant No. 01-02-16306 of the Russian Foundation for Basic Research.

\section{References}

Allen, C. W. 1973, Astrophysical Quantities (Athlone Press, London)

van Altena, W. F. 1969, AJ, 74, 2

Duflot, M., Figon, P., \& Meyssonnier, N. 1995, A\&AS, 114, 269

Eggen, O. J. 1998, AJ, 116, 782

ESA 1997, The Hipparcos and Tycho catalogues, ESA SP-1200

Kholopov, P. N. 1981, Stellar Clusters. Nauka, Moscow, in Russian

Roman, N. G. 1949, ApJ, 110, 205

Röser, S., \& Bastian, U. 1991, PPM Star Catalogue, vol. I and II, Astronomisches Rechen Institut, Heidelberg

Sachs, L. 1972, Statistische Auswertungsmetoden (SpringerVerlag)

Soderblom, D. R., \& Mayor, M. 1993, AJ, 105, 226

Urban, S. E., Corbin, T. E., \& Wycoff, G. L. 1998, AJ, 115, 2161 